\title{
From immune homeostasis to inflammation, a question of rhythms
}

Chloé C. Nobis ${ }^{1,2}$, Nathalie Labrecque ${ }^{2}$ and Nicolas Cermakian ${ }^{1 *}$

${ }^{1}$ Douglas Mental Health University Institute, McGill University, Montreal, QC H4H 1R3, Canada

${ }^{2}$ Maisonneuve-Rosemont Hospital Research Centre, University of Montreal, Montreal, QC H1T 2M4, Canada

*Corresponding author: nicolas.cermakian@mcgill.ca 


\begin{abstract}
Numerous physiological processes vary according to the time of the day in mammals, including the immune defense mechanisms. These circadian rhythms are generated by circadian clocks located in most cell types including cells of the immune system. Recent research has shown that circadian clock function is key to maintaining the homeostasis of the immune system. Indeed, the development and differentiation of different immune cells, and their trafficking between tissues, rely on proper clock function. Disruption of immune system homeostasis can lead to the development of pathological processes such as inflammation or autoimmune diseases. Circadian clocks also control the response of the immune system to inflammatory challenges and infections, and clock dysfunction can provoke or enhance disease. This review focuses on the role of circadian clocks in the homeostasis of immune cells, such as cell recirculation in the peripheral blood and tissues, as well as in the context of inflammation in response to infectious challenges or autoimmune disorders.
\end{abstract}

Keywords: Circadian clock, clock genes, immune response, inflammation, infection, autoimmunity. 


\section{Introduction: circadian clocks in the immune system}

Circadian clocks are composed of clock genes, such as Clock, Bmall, Per1-3, Cryl-2, and Reverb/Ror genes, expressed in nearly all cells and tissues in mammals [1]. The clock genes and their protein products function within transcriptional-translational feedback loops [1]. As a consequence of these feedback loops, clock transcription factors such as CLOCK/BMAL1 and REV-ERB $\alpha$ display an activity that varies over the $24 \mathrm{~h}$ day, and thus, numerous target genes of these factors display circadian rhythms in their expression levels. Such mechanisms also occur within cells of the immune system. Therefore, a circadian variation of various aspects of the innate and the adaptive immune system has been observed in mammals, both at steady state and upon immune challenges, such as infection with pathogens or inflammatory conditions.

Clock genes are expressed in most cell types of the innate and adaptive arms of the immune system [2-4]. Within innate immunity (the first lines of defense against non-self-bodies), basophils [5], eosinophils [6], mast cells [6-8], monocytes/macrophages [9-13], dendritic cells [13,14], and natural killer cells [15] all express clock genes rhythmically. This is also the case for cells of the adaptive immunity (i.e. the antigen-specific immune response), $\mathrm{T}[16,17]$ and B lymphocytes [13]. Some consider intestinal epithelial cells and cells lining the lung bronchiole as "non-conventional" immune cells, which were also found to express clock genes $[18,19]$. Thus, over the past decade work in the emerging field of chronoimmunology has highlighted the relevance of circadian rhythms in the regulation of the immune system to confer an evolutionary advantage to the organism in the context of environmental cycles of lighting, temperature, pathogen encounter, etc. This review will provide an overview of recent findings on the contributions of circadian clocks to the homeostasis and responses of the immune system.

\section{Circadian clocks and immune cell development and differentiation}

The expression of molecular clock components by most immune cells raises the question of whether the circadian clock in these cells is important for their development and function. A few studies have shown 
that indeed, the clock is required for successful immune cell development and differentiation. This is the case of the T helper 17 (Th17) cells [20] and innate lymphoid cells [21]. In both cases the mechanism relies on a rhythmic transcriptional repressor called Nuclear Factor Interleukin 3-Regulated (NFIL3, also known as E4 Promoter-Binding Protein 4, E4BP4). NFIL3 is known to bind D-box promoter elements, where it competes with PAR transcription activators such as DBP [2]. As for all rhythmically-expressed transcription factors, NFIL3 can generate circadian rhythms of expression of its gene targets [2]. Within T cells, REV-ERB $\alpha$ (a transcription factor whose expression is activated by CLOCK/BMAL1) represses the expression of NFIL3. In turn, NFIL3 represses the expression of ROR $\gamma \mathrm{t}$, the master transcriptional regulator of Th17 cell differentiation. Accordingly, Th17 counts (following CD4 T cell polarization) are higher when using cells of Nfil3 KO mice and lower with cells from Rev-erb $\alpha$ KO mice. Thus, NFIL3 can be viewed as a suppressor of Th17 cell differentiation [20]. A follow up study demonstrated a role for NFIL3 in the development of innate lymphoid cells from their precursors [20].

Whereas the differentiation of specific T cell subtypes may be relying on their endogenous clock, two studies highlighted that the development of $\mathrm{T}$ cells from progenitors is not affected by their own intrinsic clock. Indeed, in mice with a T cell-specific deletion of the essential clock gene Bmal1 [17], as well as in irradiated wild-type mice grafted with Bmal1 KO bone marrow [22], similar CD4 and CD8 T cell counts were found compared to the respective control mice.

In constrast, the development of B cells, which are involved in the humoral immune response, appears to be controlled by the circadian clock [22]. Indeed, in Bmall KO mice, the number of fully developed B cells is lower than in WT mice [22]. Interestingly though, bone marrow chimera experiments mice showed that the need for clock function for cell development is not intrinsic to the B cells themselves, but is rather in the non-hematopoietic cells [22]. This is in line with data on B cell-specific Bmall KO mice, showing intact B cells counts compared to wild-type littermates [17]. 
In sum, T cell and B cell development appears insensitive to disruption of the clock in these cells or their progenitors. However, the subsequent differentiation of these cells in specific subsets might be controlled by their endogenous clocks, as illustrated by the studies on Th17 cell differentiation.

\section{Circadian clocks and immune cell trafficking}

Immune cells recirculate to the different secondary lymphoid organs via the bloodstream and the lymph. A number of recent studies have uncovered a role for circadian clocks in regulating this immune cell trafficking. The blood and spleen counts of inflammatory monocytes present a daily rhythm in mice, with a maximum in the daytime and a minimum at night (Figure 1), and an antiphasic rhythm in the bone marrow [12]. This rhythm appears to be dependent on the clock within these monocytes, as it is lost in mice KO for the essential clock gene Bmall specifically in myeloid cells (Figure 1). The data from this study, and from other reports [11,23], suggest that the clock in myeloid cells controls the circadian expression of C-C Motif Chemokine Ligand 2 (CCL2), a chemokine important for the trafficking of monocytes.

Clock control of adaptive immune cell trafficking has also been studied. T and B lymphocytes recirculate in a circadian manner in the blood and lymphoid organs [24-27]. Prior work has shown that the recruitment of white blood cells to tissues was regulated by the autonomic nervous system and adrenergic signaling, themselves under the control of the central circadian clock in the brain [28]. Several recent studies have started to uncover the mechanisms for the circadian control of $\mathrm{T}$ cell trafficking. Suzuki et al. found a rhythm of T cell counts in lymph nodes (LNs) and showed an involvement of the adrenergic pathways: the day-night difference of $\mathrm{T}$ and $\mathrm{B}$ cell counts in LNs, and the variation of lymphocyte egress from these organs, were lost after depletion of adrenergic nerves by treatment of mice with 6-OHDA, and in mice $\mathrm{KO}$ for the $\beta_{2}$ adrenergic receptor [26]. In parallel, Druzd et al. also studied the rhythm of $\mathrm{T}$ cell counts in the LNs, but they identified a distinct mechanism [27]. Using mice lacking clock function specifically in T cells, they showed that the T cell clock was essential for the LN T cell 
count rhythm by regulating both the entrance into and exit from these organs (Figure 1). It does so by inducing rhythms of the expression of Sphingosine-1-phosphate receptor 1 (S1PR1) and C-C Motif Chemokine Receptor 7 (CCR7), two receptors important for T cell trafficking in the LN [27].

A more recent study shed new light on the T cell trafficking rhythm to lymphoid organs. Shimba et al. showed that in mice, glucocorticoids, via their receptor, act on the expression of IL-7 receptor, which varies as a function of time of day at the surface of T cells [29]. This in turns leads to a rhythm of the expression of C-X-C chemokine receptor 4 (CXCR4), which is involved in the recruitment of T cells to lymphoid organs. A parallel can be made with earlier studies in humans, which have also linked cortisol secretion with CXCR4 expression and blood T cell counts [30,31]. Indeed, these studies showed a negative correlation between $\mathrm{T}$ cell numbers and cortisol concentration in the blood. Both the endogenous morning cortisol rise and exogenous cortisol administration decreased $\mathrm{T}$ cell blood counts, and cortisol increased CXCR4 levels at the surface of T cells.

In humans, blood $\mathrm{T}$ cell counts exhibit a peak during the night while in mice, the peak is in the daytime $[24,25,32]$. Both the glucocorticoid rhythm in the blood and the rhythm of CXCR4 in T cells show an opposite phase in humans (diurnal) vs. mice (nocturnal). This opposite phase of glucocorticoids and CXCR4 might explain the opposite phases of T cell counts in humans vs. mice. However, Zhao et al. have recently proposed another mechanism. They used humanized mice, which were transplanted with human hematopoietic stem cells [33]. Thus, within the same animals, they were able to compare blood counts and protein expression for cells of both human and mouse origin. White blood cell count rhythms and CXCR4 expression rhythms had opposite phases in the mouse and human cells within these humanized mice, which is consistent with the previously described studies. However, Zhao et al suggested a new possible mechanism for the circadian regulation of CXCR4 expression that involves reactive oxygen species (ROS) [33]. In this model, the opposite rhythms in mice and humans would be due to an opposite effect of MAP kinase activation on ROS generation. However, the lack of rhythms of 
clock gene expression in the human white blood cells suggests that in this model, they might not be receiving the proper cues from the mouse environment, which may affect their trafficking.

\section{Circadian clocks and the inflammatory response to microbe-derived molecules}

The innate immune system constitutes the first line of defense against infections. As innate immune cells express the clock machinery, it is not a surprise that their responses are controlled in a circadian fashion. Innate immune cells recognize pathogens via receptors for molecules named pathogenassociated molecular patterns (PAMPs). These receptors, called pattern recognition receptors (PRR), were in some cases shown to have a daily rhythm of expression in mice. This is the case for Toll-like Receptor 5 (TLR5), which recognizes bacterial flagellin. This triggers the activation of the NFkB pathway, involved in stimulating proinflammatory cytokine secretion [34]. In fact, CLOCK protein interacts with the p65 subunit of NFאB and enhances its transcriptional activity on target genes including inflammatory cytokines [34]. TLR9 recognizes bacterial and viral DNA. The expression levels of this receptor fluctuate over the $24 \mathrm{~h}$ cycle in mouse peritoneal macrophages. These levels are correlated with the magnitude of the response to vaccination in the presence of a TLR9 ligand as adjuvant, with a maximum of the response around the middle of the night [35].

Macrophages can produce proinflammatory cytokines such as interleukin(IL)-1 $\beta$, IL-6, TNF $\alpha$, in response to PAMPs. Several groups have demonstrated a circadian control of cytokine secretion by macrophages in response to the bacterial endotoxin lipopolysaccharide (LPS), both in mice and using cells ex vivo, with a higher secretion at the day/night transition [10-12,36,37]. This rhythmic regulation appears to be cell-autonomous (i.e. relying on the clock in macrophages), because a myeloid-specific KO of Bmall abolished the morning/evening difference, with morning levels of cytokine response being increased to the evening levels $[11,36]$. The receptor for LPS is TLR4. A transcriptomic study on peritoneal macrophages showed a rhythm of transcripts for signaling molecules downstream of TLR4 
[10]. Moreover, a recent proteomic study on Kupffer cells (the liver-resident macrophages) showed a 24 $\mathrm{h}$ rhythm of TLR4 protein (and downstream signaling molecules), with a peak during the early daytime [38]. Interestingly, the relationship between the macrophage clock and LPS response is bidirectional, as the LPS- and NFkB-induced microRNA mir-155 was shown to act directly on BMAL1 expression. This pathway leads to a suppression of Bmal1 expression in macrophages upon LPS stimulation, which in turn leads to an increased secretion of pro-inflammatory cytokines [36]. Other studies pointed to an impact of Per2 and Cry gene mutation on the response to LPS [39,40]. Environmental circadian disruption can also affect the response to LPS. Indeed, simulated shift work in rats and repeated jet lag in mice led to an increase of the inflammatory responses after an LPS challenge [41,42]. In humans, it was shown that the circadian rhythm of response of monocytes to LPS became desynchronized from the rhythm of blood monocyte counts in subjects placed in a schedule simulating night shift work [32]. This showed that the regulation of these two rhythms depends on distinct mechanisms, and that abnormal sleep-wake timing can impact immune functions in humans.

Other TLRs were recently added to the list of PRRs showing rhythmic expression and/or function. The liver macrophage proteomic study already cited above also showed a daily rhythm of TLR3 and TLR8 in liver-resident macrophages [38]. A recent study provided evidence that a day-night difference in the severity of sepsis upon cecal ligation and puncture in mice relies on TLR2 (but not TLR9), and on the clock in leukocytes [43]. Silver and colleagues looked at the expression and function of many TLRs. All the PRRs they tested (TLR1-8) showed time of day-dependent mRNA expression in spleen cells, and some of their responses to their respective PAMPs showed time of day-dependence [44]. They also showed a diurnal rhythm of TLR3 response in vivo.

These studies confirmed a role of the circadian machinery in the cytokine response to bacterial endotoxin challenge. What is the molecular link between the clock and the cytokine response? Many studies from the past few years have pointed to REV-ERB $\alpha$ as a major intermediate. Mice KO for Rev- 
erb $\alpha$ and human cells with shRNA against this clock gene both show increased IL-6 response to LPS, and conversely, overexpressing REV-ERB $\alpha$ or stimulating its activity with a specific ligand blunted LPS-induced IL-6 expression and release [11,23]. REV-ERB $\alpha$ achieves a circadian expression of macrophage-specific genes by binding to enhancers selected by macrophage lineage-determining factors [45]. A similar role of REV-ERB $\alpha$ in modulating the inflammatory response was also found in a pulmonary infection model, in which LPS is aerosolized into the airway of mice. The inflammatory response to LPS inhalation follows a circadian rhythm, including a circadian rhythm of the production of the CXCL5 chemokine by the bronchiole epithelial cells, and consequently, of the recruitment of neutrophils to the lungs [18]. A recent study showed that in mice KO for Rev-Erb $\alpha$ (either globally or specifically in the lung epithelial cells), there was an increased inflammatory response to LPS (with increased IL-6 and CXCL5 expression) and a loss of the rhythm of neutrophil infiltration in the lung [46]. Again, a REV-ERB $\alpha$ agonist led to a suppression of the IL-6 response [46].

Recently, a study from Pourcet and colleagues provided a mechanism for the action of REV-ERB $\alpha$ on the inflammasome, which is important for the response to non-infectious danger-associated molecular patterns [47]. The activation of the NLRP3 inflammasome is critical for the maturation and secretion of cytokines such as IL-1 $\beta$ and IL-18. They showed that REV-ERB $\alpha$ directly regulates the expression of the Nlrp3 gene, encoding the core component of the NLRP3 inflammasome. Thus, REV-ERB $\alpha$, via the repression of Nlrp3 but also by a direct action on the $I l-1 \beta$ and $I l-18$ genes, leads to a rhythm of NLRP3 inflammasome activity and cytokine expression. The study also shows the impact of this regulation in mouse models of peritonitis and fulminant hepatitis, in which REV-ERB $\alpha$ has a protective antiinflammatory effect at certain times of the day via regulation of NLRP3 expression [47].

\section{Circadian clocks and the control of infections}


Over the past few years, studies have started to show the importance of circadian clocks for the control of different types of infection (Figure 2). This is the case for infection of mice with the bacteria Diplococcus pneumoniae [48] and Salmonella enterica serovar Typhimurium[37]: a day/night variation was found, with a higher mouse mortality or bacterial load after infection during the daytime compared to the night time. Moreover, in the case of Salmonella, a corresponding rhythm of mRNA expression of inflammation markers was observed in the colon [37]. These daily variations were abolished in Clock mutant mice, confirming a role of the clock in the control of the Salmonella infection. The clocks in immune cells also underlie a rhythm of the outcome of infection with the bacterium Listeria monocytogenes, with higher pathogenicity (and poorer mouse survival) in the second half of the light period vs. in the early day [12].

Two studies have shown an effect of the circadian clock on viral infections. Edgar and colleagues showed that the level of viral replication upon infection of mice with herpesvirus varies according to the time of time, a variation that is abolished (and overall levels increased) in Bmall KO mice [49]. Another group showed that the inflammatory response, encephalitis and survival of mice following vesicular stomatitis virus infection was dependent on the time of infection, and that the clock protein REV-ERB $\alpha$ affects disease outcome [50]. One study has recently shown a time-dependent variation of the control of infection by the fungus Aspergillus fumigatus [51].

Finally, an implication of circadian clocks was studied in the context of parasitic infections. Indeed, infection of mice with the protozoan parasite Leishmania major leads to a higher parasite load when done in the early night compared to infection in the early day, both upon infection in the footpad or intraperitoneally (i.p.) [52]. This can be put in parallel with a higher inflammatory response upon i.p. infection in the early night compared to the early day, both in terms of recruitment of neutrophils and macrophages (the cells that act as hosts for Leishmania) and chemokine/cytokine expression. All these rhythms were abolished in mice with clock dysfunction in hematopoietic cells [52]. Another group studied infection with the intestinal worm parasite Trichuris muri. The expulsion of the parasite and the cytokine response 
depended on the time of infection [14]. Interestingly, mice with a dendritic cell (DC)-specific Bmall deletion lost the morning/evening difference in worm burden and cytokine secretion, showing the importance of the DC clock in the development of the response to the infection [14]. The case of parasitic infections is interesting in that it raises the question of the contribution of circadian control in parasitehost interactions. The parasite has rhythms as well as the transmission vector (e.g. the sandfly in the case of Leishmania), and how this is coordinated with the host's immune system's rhythms has implications both for understanding the evolution of parasite-host interactions and to design new prophylactic strategies [53]. This is especially important in the context of the recent discovery of endogenous circadian rhythms of gene expression in the protozoan parasite Trypanosoma brucei, which suggests that it has its own circadian clock, although its molecular components have yet to be defined [54]. The synchrony between the parasite and the host was addressed for Plasmodium infection (Plasmodium is the causing agent of malaria). Plasmodium infection (in its red blood cell stage) displays a daily ( $24 \mathrm{~h}$ or a multiple of $24 \mathrm{~h}$ ) rhythmicity, synchronous among red blood cells. Infection with parasite-bearing red blood cells of mice with a matched or reversed light:dark cycle was compared: parasites out of synchrony with the host had reduced proliferation and transmission potential [55].

\section{Circadian clocks in animal models of autoimmune disorders}

In autoimmune diseases, the immune system attacks self tissues. Given that circadian clocks regulate immunity, a deregulation of this rhythmic regulation could conceivably lead to excessive immune responses, and autoimmunity. Therefore, several recent studies have tested the impact of circadian clocks in animal models of autoimmune diseases. Rheumatoid arthritis (RA) is an autoimmune disease involving inflammation in the joints, and overgrowth of fibroblast-like synovial cells, and joint remodeling and destruction. Symptoms of RA display daily rhythms, being increased in the morning [56]. Experimental arthritis consists in injecting rodents with an antibody against type II collagen, or to immunize the animals with type II collagen and an immune adjuvant. Clock genes are expressed in 
cultured human rheumatoid synovial cells [57] and in mouse synovial cells [58,59]. In the joints of rodents with experimental arthritis, clock gene expression is altered, and the extent of disease varies according to the time of arthritis-inducing treatment. Moreover, clock disruption by constant light or clock gene deletion or knockdown led to altered clock gene expression in the joints, exacerbated joint inflammation, and increased arthritis score $[58,59]$.

Another autoimmune disorder that has been studied in the context of circadian clocks is multiple sclerosis (MS). In MS patients, the immune system attacks myelin in the central nervous system. Experimental autoimmune encephalomyelitis (EAE) is an experimental model of MS where mice are immunized with $\mathrm{MOG}_{35-55}$ (a peptide antigen consisting in a fragment of a myelin protein) together with Freund's adjuvant. This leads to a demyelination of neurons similar to that occurring in MS patients. Two reports showed the importance of the circadian clocks of both $\mathrm{T}$ cells and myeloid cells for disease progression in EAE mice (Figure 3). Day/night differences were observed, with higher clinical score after $\mathrm{MOG}_{35-55}$ immunization in the daytime. These day/night differences were abolished both in $\mathrm{T}$ cellspecific and myeloid cell-specific Bmall KO mice, with the higher daytime disease score going down to the lower nighttime level, indicating a protective effect of the mutation [27,60]. It will be interesting to understand the mechanisms for the respective involvement of these two distinct circadian clocks in the control of the disease in MS. Interestingly, in humans, MS relapses showed a variation according to the time of year, with more cases during spring and summer, compared to the rest of the year. This was inversely correlated with levels of the circadian hormone melatonin. Using human cells and mice, it was found that melatonin affects the differentiation of cell types with opposite roles in MS: it inhibits the differentiation into Th17 (via NFIL3), which contribute to the pathology, whereas it promotes the production of regulatory $\mathrm{T}$ (Treg) cells, which are protective [61].

\section{Conclusion}


In this review, we mainly focused on the circadian control of innate arm of the immune system. The recent studies presented here highlight the importance of circadian clocks both in the control of the homeostasis and when the organism faces an infectious challenge. Recent work has started to point to a circadian control of the adaptive immune response too (beyond the $\mathrm{T}$ cell trafficking aspect described in the present review). Moreover, it will be necessary to understand the molecular mechanisms underlying the circadian control of immunity, to better define how to act on these pathways to design new therapies.

\section{Acknowledgements}

This work was supported by a grant from the Canadian Institutes for Health Research (MOP119322, to NC and NL).

Declarations of interest: none. 


\section{Figure legends}

\section{Figure 1. Circadian rhythms of immune cell trafficking}

Profiles of the rhythm of immune cell trafficking in the blood and lymphoid organs in mice. Inflammatory monocytes are shown for wild-type (thick lines) and myeloid-specific Bmall knock-out mice (dotted lines). T lymphocytes are shown for wild-type (thick lines) and T lymphocyte-specific Bmall knock-out mice (dotted line).

\section{Figure 2. Circadian control of the infectious disease development}

Circadian clocks regulate antiviral responses (in blue), anti-bacterial responses (in red), and responses to eukaryotic pathogens (in green) in mouse models. As a result, the pathogen load, the immune response and the host's survival are all susceptible to vary according to the time of day of initial infection. The right part of the figure displays the outcome of infections occurring in the daytime (or subjective day for experiments in constant darkness). The left part of the figure displays the outcome of infections occurring in the night time (or subjective night for experiments in constant darkness). The numbers are Zeitgeber times (hours after the onset of light in the morning, under a light:dark cycle) or Circadian times (corresponding times under constant darkness).

Figure 3. Control of the severity of experimental autoimmune encephalomyelitis (EAE) and of immune cell counts by the circadian clock

Day (left, yellow) and night (right, grey) levels of immune cells in wild-type mice (top) and cell typespecific Bmal1 knock-out mice (bottom). Figure insets present a very simplified view of the clock feedback loop and graph insets present the time-dependence of the EAE clinical score in wild-type (WT) mice (top) and the lack of time-dependence in cell type-specific Bmall knock-out KO) mice (bottom). T lymphocyte counts are shown for experiments comparing WT vs. T lymphocyte-specific Bmall KO 
mice, whereas neutrophil and monocyte counts are shown for experiments comparing WT vs. mice for Bmall KO in myeloid cells and granulocytes (LysM-Cre). 


\section{References}

1. Duguay D, Cermakian N: The crosstalk between physiology and circadian clock proteins. Chronobiol Int 2009, 26:1479-1513.

2. Curtis AM, Bellet MM, Sassone-Corsi P, O'Neill LA: Circadian clock proteins and immunity. Immunity 2014, 40:178-186.

3. Labrecque N, Cermakian N: Circadian Clocks in the Immune System. J Biol Rhythms 2015, 30:277-290.

4. Nobis CCL, N; Cermakian, N.: Circadian control of antigen-specific T cell responses. ChronoPhysiology and Therapy 2016, 6:65-74.

5. Ando N, Nakamura Y, Ishimaru K, Ogawa H, Okumura K, Shimada S, Nakao A: Allergen-specific basophil reactivity exhibits daily variations in seasonal allergic rhinitis. Allergy 2015, 70:319-322.

6. Baumann A, Gonnenwein S, Bischoff SC, Sherman H, Chapnik N, Froy O, Lorentz A: The circadian clock is functional in eosinophils and mast cells. Immunology 2013, 140:465-474.

7. Nakamura Y, Ishimaru K, Tahara Y, Shibata S, Nakao A: Disruption of the suprachiasmatic nucleus blunts a time of day-dependent variation in systemic anaphylactic reaction in mice. J Immunol Res 2014, 2014:474217.

8. Nakamura Y, Nakano N, Ishimaru K, Hara M, Ikegami T, Tahara Y, Katoh R, Ogawa H, Okumura $\mathrm{K}$, Shibata S, et al.: Circadian regulation of allergic reactions by the mast cell clock in mice. J Allergy Clin Immunol 2014, 133:568-575.

9. Hayashi M, Shimba S, Tezuka M: Characterization of the molecular clock in mouse peritoneal macrophages. Biol Pharm Bull 2007, 30:621-626. 
10. Keller M, Mazuch J, Abraham U, Eom GD, Herzog ED, Volk HD, Kramer A, Maier B: A circadian clock in macrophages controls inflammatory immune responses. Proc Natl Acad Sci U S A 2009, 106:21407-21412.

11. Gibbs JE, Blaikley J, Beesley S, Matthews L, Simpson KD, Boyce SH, Farrow SN, Else KJ, Singh D, Ray DW, et al.: The nuclear receptor REV-ERBalpha mediates circadian regulation of innate immunity through selective regulation of inflammatory cytokines. Proc Natl Acad Sci U S A 2012, 109:582-587.

12. Nguyen KD, Fentress SJ, Qiu Y, Yun K, Cox JS, Chawla A: Circadian gene Bmal1 regulates diurnal oscillations of Ly6C(hi) inflammatory monocytes. Science 2013, 341:1483-1488. 13. Silver AC, Arjona A, Hughes ME, Nitabach MN, Fikrig E: Circadian expression of clock genes in mouse macrophages, dendritic cells, and B cells. Brain Behav Immun 2012, 26:407-413.

14. Hopwood TW, Hall S, Begley N, Forman R, Brown S, Vonslow R, Saer B, Little MC, Murphy EA, Hurst RJ, et al.: The circadian regulator BMAL1 programmes responses to parasitic worm infection via a dendritic cell clock. Sci Rep 2018, 8:3782.

15. Arjona A, Sarkar DK: Circadian oscillations of clock genes, cytolytic factors, and cytokines in rat NK cells. J Immunol 2005, 174:7618-7624.

16. Bollinger T, Leutz A, Leliavski A, Skrum L, Kovac J, Bonacina L, Benedict C, Lange T, Westermann J, Oster H, et al.: Circadian clocks in mouse and human CD4+ T cells. PLoS One 2011, 6:e29801.

17. Hemmers S, Rudensky AY: The Cell-Intrinsic Circadian Clock Is Dispensable for Lymphocyte Differentiation and Function. Cell Rep 2015, 11:1339-1349.

18. Gibbs J, Ince L, Matthews L, Mei J, Bell T, Yang N, Saer B, Begley N, Poolman T, Pariollaud M, et al.: An epithelial circadian clock controls pulmonary inflammation and glucocorticoid action. Nat Med 2014, 20:919-926. 
19. Wang Y, Kuang Z, Yu X, Ruhn KA, Kubo M, Hooper LV: The intestinal microbiota regulates body composition through NFIL3 and the circadian clock. Science 2017, 357:912-916.

20. Yu X, Rollins D, Ruhn KA, Stubblefield JJ, Green CB, Kashiwada M, Rothman PB, Takahashi JS, Hooper LV: TH17 cell differentiation is regulated by the circadian clock. Science 2013, 342:727-730.

21. Yu X, Wang Y, Deng M, Li Y, Ruhn KA, Zhang CC, Hooper LV: The basic leucine zipper transcription factor NFIL3 directs the development of a common innate lymphoid cell precursor. Elife 2014, 3.

22. Sun Y, Yang Z, Niu Z, Peng J, Li Q, Xiong W, Langnas AN, Ma MY, Zhao Y: MOP3, a component of the molecular clock, regulates the development of B cells. Immunology 2006, 119:451-460.

23. Sato S, Sakurai T, Ogasawara J, Takahashi M, Izawa T, Imaizumi K, Taniguchi N, Ohno H, Kizaki T: A circadian clock gene, Rev-erbalpha, modulates the inflammatory function of macrophages through the negative regulation of Ccl2 expression. J Immunol 2014, 192:407417.

24. Born J, Lange T, Hansen K, Molle M, Fehm HL: Effects of sleep and circadian rhythm on human circulating immune cells. J Immunol 1997, 158:4454-4464.

25. Kirsch S, Thijssen S, Alarcon Salvador S, Heine GH, van Bentum K, Fliser D, Sester M, Sester U: T-cell numbers and antigen-specific T-cell function follow different circadian rhythms. $J$ Clin Immunol 2012, 32:1381-1389.

26. Suzuki K, Hayano Y, Nakai A, Furuta F, Noda M: Adrenergic control of the adaptive immune response by diurnal lymphocyte recirculation through lymph nodes. J Exp Med 2016, 213:2567-2574. 
27. Druzd D, Matveeva O, Ince L, Harrison U, He W, Schmal C, Herzel H, Tsang AH, Kawakami N, Leliavski A, et al.: Lymphocyte Circadian Clocks Control Lymph Node Trafficking and Adaptive Immune Responses. Immunity 2017, 46:120-132.

28. Scheiermann C, Kunisaki Y, Lucas D, Chow A, Jang JE, Zhang D, Hashimoto D, Merad M, Frenette PS: Adrenergic nerves govern circadian leukocyte recruitment to tissues. Immunity 2012, 37:290-301.

29. Shimba A, Cui G, Tani-Ichi S, Ogawa M, Abe S, Okazaki F, Kitano S, Miyachi H, Yamada H, Hara T, et al.: Glucocorticoids Drive Diurnal Oscillations in T Cell Distribution and Responses by Inducing Interleukin-7 Receptor and CXCR4. Immunity 2018, 48:286-298 e286.

30. Dimitrov S, Benedict C, Heutling D, Westermann J, Born J, Lange T: Cortisol and epinephrine control opposing circadian rhythms in T cell subsets. Blood 2009, 113:5134-5143.

31. Besedovsky L, Linz B, Dimitrov S, Groch S, Born J, Lange T: Cortisol increases CXCR4 expression but does not affect CD62 $\mathrm{L}$ and CCR7 levels on specific $\mathrm{T}$ cell subsets in humans. Am J Physiol Endocrinol Metab 2014, 306:E1322-1329.

32. Cuesta M, Boudreau P, Dubeau-Laramee G, Cermakian N, Boivin DB: Simulated Night Shift Disrupts Circadian Rhythms of Immune Functions in Humans. J Immunol 2016, 196:24662475.

33. Yue Zhao ML, Xue Ying Chan, Sue Yee Tan, Sharrada Subramaniam, Yong Fan, Eva Loh,, Kenneth Tou En Chang TCT, and Qingfeng Chen: Uncovering the mystery of opposite circadian rhythms between mouse and human leukocytes in humanized mice. IMMUNOBIOLOGY AND IMMUNOTHERAPY 2018, 130:1995-2005.

34. Spengler ML, Kuropatwinski KK, Comas M, Gasparian AV, Fedtsova N, Gleiberman AS, Gitlin, II, Artemicheva NM, Deluca KA, Gudkov AV, et al.: Core circadian protein CLOCK is a 
positive regulator of NF-kappaB-mediated transcription. Proc Natl Acad Sci U S A 2012, 109:E2457-2465.

35. Silver AC, Arjona A, Walker WE, Fikrig E: The circadian clock controls toll-like receptor 9mediated innate and adaptive immunity. Immunity 2012, 36:251-261.

36. Curtis AM, Fagundes CT, Yang G, Palsson-McDermott EM, Wochal P, McGettrick AF, Foley NH, Early JO, Chen L, Zhang H, et al.: Circadian control of innate immunity in macrophages by miR-155 targeting Bmal1. Proc Natl Acad Sci U S A 2015, 112:7231-7236.

37. Bellet MM, Deriu E, Liu JZ, Grimaldi B, Blaschitz C, Zeller M, Edwards RA, Sahar S, Dandekar S, Baldi P, et al.: Circadian clock regulates the host response to Salmonella. Proc Natl Acad Sci U S A 2013, 110:9897-9902.

38. Wang Y, Song L, Liu M, Ge R, Zhou Q, Liu W, Li R, Qie J, Zhen B, Wang Y, et al.: A proteomics landscape of circadian clock in mouse liver. Nat Commun 2018, 9:1553.

39. Liu J, Malkani G, Shi X, Meyer M, Cunningham-Runddles S, Ma X, Sun ZS: The circadian clock Period 2 gene regulates gamma interferon production of NK cells in host response to lipopolysaccharide-induced endotoxic shock. Infect Immun 2006, 74:4750-4756.

40. Narasimamurthy R, Hatori M, Nayak SK, Liu F, Panda S, Verma IM: Circadian clock protein cryptochrome regulates the expression of proinflammatory cytokines. Proc Natl Acad Sci U S A 2012, 109:12662-12667.

41. Guerrero-Vargas NN, Guzman-Ruiz M, Fuentes R, Garcia J, Salgado-Delgado R, Basualdo Mdel C, Escobar C, Markus RP, Buijs RM: Shift Work in Rats Results in Increased Inflammatory Response after Lipopolysaccharide Administration: A Role for Food Consumption. J Biol Rhythms 2015, 30:318-330.

42. Castanon-Cervantes O, Wu M, Ehlen JC, Paul K, Gamble KL, Johnson RL, Besing RC, Menaker M, Gewirtz AT, Davidson AJ: Dysregulation of inflammatory responses by chronic circadian disruption. J Immunol 2010, 185:5796-5805. 
43. Heipertz EL, Harper J, Lopez CA, Fikrig E, Hughes ME, Walker WE: Circadian Rhythms Influence the Severity of Sepsis in Mice via a TLR2-Dependent, Leukocyte-Intrinsic Mechanism. J Immunol 2018, 201:193-201.

44. Silver AC, Buckley SM, Hughes ME, Hastings AK, Nitabach MN, Fikrig E: Daily oscillations in expression and responsiveness of Toll-like receptors in splenic immune cells. Heliyon 2018, 4:e00579.

45. Lam MT, Cho H, Lesch HP, Gosselin D, Heinz S, Tanaka-Oishi Y, Benner C, Kaikkonen MU, Kim AS, Kosaka M, et al.: Rev-Erbs repress macrophage gene expression by inhibiting enhancer-directed transcription. Nature 2013, 498:511-515.

46. Pariollaud M, Gibbs J, Hopwood T, Brown S, Begley N, Vonslow R, Poolman T, Guo B, Saer B, Jones DH, et al.: Circadian clock component REV-ERBalpha controls homeostatic regulation of pulmonary inflammation. J Clin Invest 2018, 128(6):2281-2296.

47. Pourcet B, Zecchin M, Ferri L, Beauchamp J, Sitaula S, Billon C, Delhaye S, Vanhoutte J, MayeufLouchart A, Thorel Q, et al.: Nuclear Receptor Subfamily 1 Group D Member 1 Regulates Circadian Activity of NLRP3 Inflammasome to Reduce the Severity of Fulminant Hepatitis in Mice. Gastroenterology 2018, 154:1449-1464 e1420.

48. Shackelford PG, Feigin RD: Periodicity of susceptibility to pneumococcal infection: influence of light and adrenocortical secretions. Science 1973, 182:285-287.

49. Edgar RS, Stangherlin A, Nagy AD, Nicoll MP, Efstathiou S, O'Neill JS, Reddy AB: Cell autonomous regulation of herpes and influenza virus infection by the circadian clock. Proc Natl Acad Sci U S A 2016, 113:10085-10090.

50. Gagnidze K, Hajdarovic KH, Moskalenko M, Karatsoreos IN, McEwen BS, Bulloch K: Nuclear receptor REV-ERBalpha mediates circadian sensitivity to mortality in murine vesicular stomatitis virus-induced encephalitis. Proc Natl Acad Sci U S A 2016, 113:5730-5735. 
51. Chen S, Fuller KK, Dunlap JC, Loros JJ: Circadian Clearance of a Fungal Pathogen from the

Lung Is Not Based on Cell-intrinsic Macrophage Rhythms. J Biol Rhythms 2018, 33:99-105.

52. Kiessling S, Dubeau-Laramee G, Ohm H, Labrecque N, Olivier M, Cermakian N: The circadian clock in immune cells controls the magnitude of Leishmania parasite infection. Sci Rep 2017, 7:10892.

53. Martinez-Bakker M, Helm B: The influence of biological rhythms on host-parasite interactions. Trends Ecol Evol 2015, 30:314-326.

54. Rijo-Ferreira F, Pinto-Neves D, Barbosa-Morais NL, Takahashi JS, Figueiredo LM: Trypanosoma brucei metabolism is under circadian control. Nat Microbiol 2017, 2:17032.

55. O'Donnell AJ, Schneider P, McWatters HG, Reece SE: Fitness costs of disrupting circadian rhythms in malaria parasites. Proc Biol Sci 2011, 278:2429-2436.

56. Gibbs JE, Ray DW: The role of the circadian clock in rheumatoid arthritis. Arthritis Res Ther 2013, 15:205.

57. Yoshida K, Hashiramoto A, Okano T, Yamane T, Shibanuma N, Shiozawa S: TNF-alpha modulates expression of the circadian clock gene Per 2 in rheumatoid synovial cells. Scand J Rheumatol 2013, 42:276-280.

58. Hand LE, Hopwood TW, Dickson SH, Walker AL, Loudon AS, Ray DW, Bechtold DA, Gibbs JE: The circadian clock regulates inflammatory arthritis. FASEB J 2016, 30:3759-3770.

59. Hashiramoto A, Yamane T, Tsumiyama K, Yoshida K, Komai K, Yamada H, Yamazaki F, Doi M, Okamura H, Shiozawa S: Mammalian clock gene Cryptochrome regulates arthritis via proinflammatory cytokine TNF-alpha. J Immunol 2010, 184:1560-1565.

60. Sutton CE, Finlay CM, Raverdeau M, Early JO, DeCourcey J, Zaslona Z, O'Neill LAJ, Mills KHG, Curtis AM: Loss of the molecular clock in myeloid cells exacerbates $\mathbf{T}$ cell-mediated CNS autoimmune disease. Nat Commun 2017, 8:1923. 
61. Farez MF, Mascanfroni ID, Mendez-Huergo SP, Yeste A, Murugaiyan G, Garo LP, Balbuena Aguirre ME, Patel B, Ysrraelit MC, Zhu C, et al.: Melatonin Contributes to the Seasonality of Multiple Sclerosis Relapses. Cell 2015, 162:1338-1352. 
$\rrbracket$ Survival upon

Vesicular stomatitis virus infection

^ Immune response to

Murid Herpes virus 4 infection

iि Survival upon

Listeria monocytogenes infection

$\sqrt{ }$ Immune response to

Trichuris muris infection

ฯ Extent of

Aspergillus fumigatus infection

$\sqrt{ }$ Survival upon

Diplococcus pneumoniae infection

$\sqrt{ }$ Extent of

Salmonella Typhimurium infection

१ Immune response to Leishmania major infection

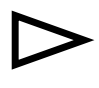

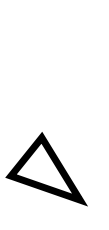

20

18

16

亿 Survival upon

Vesicular stomatitis virus infection

i Survival upon

Listeria monocytogenes infection

个 Immune response to

Trichuris muris infection

$\Downarrow$ Extent of

Aspergillus fumigatus infection $\sqrt{ }$ Immune response to

$\searrow$ Leishmania major infection

2

4

$\Delta$ Extent of Salmonella Typhimurium infection

6

8

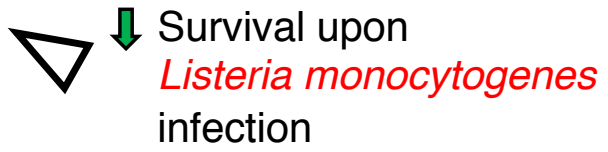

$\Downarrow$ Immune response to Murid Herpes virus 4 infection

1) Survival upon

Diplococcus pneumoniae infection 
